\title{
FAST MARKOV LOCALIZATION IN INDOOR ENVIRONMENT USING SFM ANGLE-HISTOGRAM
}

\author{
Jung-Hyun Moon ${ }^{1,2}$, Bum-Jae You ${ }^{1}$, Hagbae $\mathrm{Kim}^{2}$ and Sang-Rok Oh ${ }^{1}$ \\ ${ }^{1}$ Intelligent Robotic Research Center, Korea Institute of Science and Technology (KIST), \\ P.O.Box 131, Cheongryang, Seoul 130-650, Korea \\ ${ }^{2}$ Department of Electrical and Electronic Engineering, Yonsei University, 134, Shinchon- \\ dong, Seodaemun-ku, Seoul 120-749, Korea \\ moontey@1ycos.co.kr
}

\begin{abstract}
Localization is one of the most important issues for mobile robots since all tasks are commanded to a mobile robot based on the assumption that the mobile robot knows its position. Even though non-probabilistic techniques are faster than probabilistic approaches, those are sensitive to measurement errors and a mobile robot may lose its position in complex environments. And most simple features need additional information to represent the characteristics of environments. On the contrary, probabilistic approaches have many advantages since those can cope with sensor noises and can globally localize a mobile robot. However, those probabilistic approaches are time-consuming techniques because of the heavy computational loads due to huge comparative data. In this paper, we propose a fast probabilistic localization method including global localization by remodeling raw laser sensory data using angle histogram to reduce computational loads for localization. The algorithm is experimented successfully by using a mobile robot named KARA.
\end{abstract}

Keywords- global localization, fast probabilistic localization, sensor remodeling, angle-histgram

\section{Introduction}

Not only industrial robots are widely used, but also a number of service robots, such as vacuum cleaning robots have been introduced to commercial market. Since many tasks of mobile robots are commanded based on the assumption that each mobile robot knows its position exactly [1], localization that determines the real position of a mobile robot with respect to a world coordinate frame using sensory information and a world model for environments becomes a hot issue in the robot technology.

There are so many sources for mobile robot localizations in indoor environments. Localization algorithm can be classified into two categories according to its methodologies, non-probabilistic approaches and probabilistic approaches. For nonprobabilistic approaches, a mobile robot finds its position by feature-matching methods between model features from a reference map for environments and those from real sensory data. Vertices and lines are widely used as matching features. Vertices are the simplest features for the environments, however, it is difficult to discriminate matched vertex among other vertices because a vertex have little information except for its position. Therefore, we must have additional information that represents the environments. In [2], they use 'visibility sectors' made of order of vertices as additional information. An overall map is divided into visibility sectors using vertices of environments. After obtaining vertex data from laser range data, the position of a mobile robot is found by matching them with each visibility sectors. Unfortunately, it cannot discriminate two sectors that have the same order of vertices. In [3], they find all distances between all vertices in an overall reference map to make the reference table during pre-processing. Then, vertices are extracted by using an angle-function from real laser data and a set of distances between all obtained vertices are calculated. Finally, they find the most probable vertex set by matching the length sets in the reference table with the obtained length sets, and determine the position of a mobile. Lines are also used as matching features, however, obtaining method of reliable line information such as Generalized Hough Transform takes much time. Summing up, simple matching features need additional information for representing the characteristics of environments in most case. Therefore, it is good to find a simple feature that needs no additional information. Also, these nonprobabilistic approaches are sensitive to measurement errors, and dynamic obstacles and/or frequent changes of environments make the nonprobabilistic approaches more erroneous. So, a mobile robot may lose its position when we use nonprobabilistic approach.

For probabilistic approaches, a mobile robot finds its position by estimating its probable position from raw sensory data up to current time. Fox[4] proposes Markov localization(ML) method based on a grid map. This method can perform a global localization by updating probabilities of positions where the mobile robot can exist based on the Markov property. However, heavy computational loads for modeling of sensors and actions are required. Dellaert[5] tries to reduce the computational loads by applying Monte Carlo sampling to ML technique. Despite Monte Carlo localization method does not reduce the number of comparative sensor data, sampling of 
location candidates reduces the number of comparative positions for probability update. In practice, however, many samples more than expected are required to guarantee a good performance - such as fast convergence - for the proposed approach. Jetto and Longhi[6] used extended Kalman filter(EKF) for mobile robot localization. Using this method, a mobile robot can estimate its heading and position in spite of sensor errors. EKF is faster than ML, however, the EKF algorithm needs initial location of the mobile robot even though some errors are included. Summing up, the probabilistic approaches include heavy computational loads due to large number of comparative sensor data even though two approaches show good localization performance.

In [7], we modify Markov Localization in order to reduce overall computational loads by remodeling raw laser sensory data using angle-histogram [8]. The angle-histogram is statistical information of vector differences between two neighbor range data captured by a laser range sensor. The main advantage of using angle-histogram is that we can reduce the number of comparative data, computational loads, and calculation time finally. Another advantage is that we can find the heading angle of the mobile robot within angle resolution in a very fast manner. In [7], we update state probabilities according to the common angle-histogram. This method can reduce the comparative measurement data. However, common angle-histogram is not accurate enough to extract one grid among the area of probable grids. Also, we used no sensor model, but we use the variances between reference and obtained histogram as updating parameters. In this paper, we make the suitable-for-ML angle-histogram and change the updating procedure of ML to make angle-histogram accurate enough to find the real position exactly. Also, we reduce not only the comparative measurement data but also comparative states. Reduction of comparative states makes it faster to localize a robot.

This paper is organized as following: In section 2, we say about ML and our assumptions. In section 3, we explain our sensor remodeling and why we use angle-histogram for this method. Section 4 shows the processes of our localization algorithm. In Section 5, we examine the effectiveness of the proposed algorithm by experimental results. Finally, some conclusions and future works are outlined in Section 6.

\section{Problem Definitions}

Before starting off our algorithm, we have to define the problems that is solved in this paper and set the assumptions of the environments. For localizing a mobile robot, we use the estimation technique of the robot at current step $T$. In ML[5], the belief $\operatorname{Bel}\left(L_{t}\right)$ has to be conditioned on all previous action $a_{i}$ and percepts $s_{i}$, and we obtain the following equation which describes the belief of being at a location $l$ at time $t$ by applying Bayes' rule.

$$
\operatorname{Bel}\left(L_{t} \mid l\right)=\frac{P\left(s_{t} \mid L_{t}=l, s_{1, \cdots, t-1}, a_{1, \cdots, t-1}\right) P\left(L_{t}=l \mid s_{1, \cdots, t-1}, a_{1, \cdots, t-1}\right)}{P\left(s_{t} \mid s_{1, \cdots, t-1}, a_{1, \cdots, t-1}\right)}
$$

Dieter Fox divides this procedure into 3 parts: updating part, prediction part and normalization part. In the prediction part, we predict $P\left(L_{t} \mid L_{t-1}, a_{t-1}\right)$ by the belief of being at each previous percept and the mobile robot motion. In the updating part, author applies the assumption of 'independence of perceptions' which means that once we know $L_{t}$, all other previous measurements, prior states and actions have no influence on the prediction of $S_{t}$. Then, $P\left(S_{t} \mid L_{t}=l, S_{1, \cdots, t-1}, a_{1, \cdots, t-1}\right)$ is converted to $P\left(S_{t} \mid L_{t}\right)$. In this part, the belief of being at a location $l$ is updated by the probability calculated in the prediction part and current measurements. And we normalize the belief of all percepts in normalization part. Finally, Dieter Fox simplifies the localization equation (1) as

$$
\operatorname{Bel}\left(L_{t} \mid l\right)=\frac{P\left(s_{t} \mid l\right) P\left(L_{t} \mid L_{t-1}, a_{t-1}\right)}{P\left(s_{t} \mid L_{t}\right)}
$$

and this equation is referred as Markov update formula. Figure 1 shows the flowchart of ML.

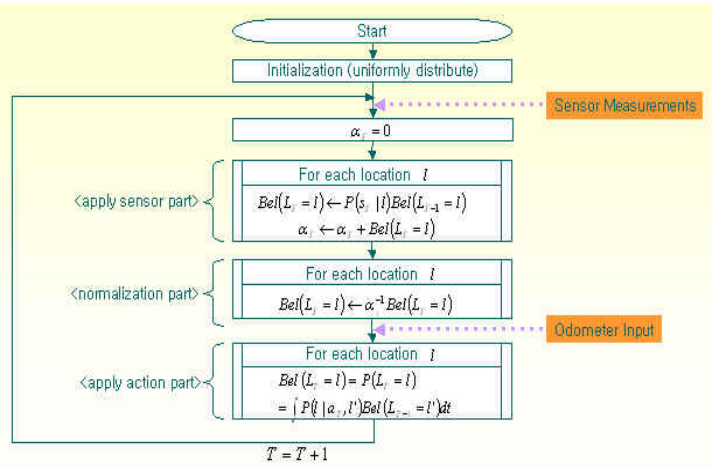

Fig.1. Flowchart of Markov Localization

In this paper, we focused on the updating part of ML. We have to perform $N_{\text {calculation }}=N_{x} \times N_{y} \times N_{\theta} \times N_{\text {data }}$ at each update step while we use $N_{x} \times N_{y}$ grid-map with $\frac{360}{N_{\theta}}$ angle resolution, and the number of laser data is $N_{\text {data }}$. For example, the size of environments is $30 \times 40(\mathrm{~m})$ and we make $300 \times 400$ grid-map(cell size of each grid is $10 \mathrm{~cm}$ ) with 2 angle resolution. And the laser range-finder covers frontal half of the robot(the number of laser data is 181), then we have to perform $3,909,600,000$ iterations at each step. Heavy calculations make it difficult to perform a task of the robot. Therefore, we reduce the number of calculations $N_{0}$ by sensor remodeling.

\subsection{Environmental Assumptions}

In this paper, our target is a service robot in indoor 
environment, and most of us work in an office and stay in an apartment. That is, a service robot works in a building. In a building, rooms and corridors are located as shown in Figure 2. That means, rooms and corridors are located along the walls, and we locate obstacles like table or cabinet parallel to walls for efficiency of area. Also, obtained laser data are point data and we regard the areas of neighbor sets of detected points as obstacles. Therefore, we can regard the environments detected by the laser rangefinder as lines. In this paper, we assume the environmental assumption as (1) large parts of the obstacles are located parallel to walls, (2) all environments are transformed into sets of lines.

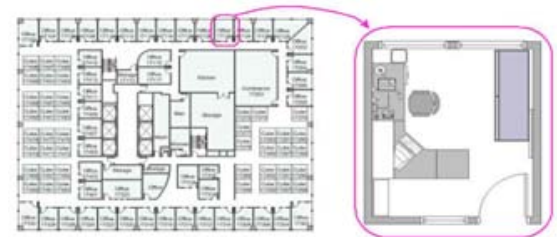

Fig.2. Example of Drawing of an Office Building

\section{Sensor Modeling}

In the probabilistic approaches including $\mathrm{ML}$, we have to perform a number of iterations to estimate the probabilities of comparative locations according to comparative measurement data per each step. Since the reduction of comparative poses of a robot makes the position estimation unreliable, we can hardly reduce the number of comparative locations even though we want to reduce the computational loads. So, we propose a new approach to reduce the number of comparative measurement data if we want to reduce the computational loads without the loss of reliability.

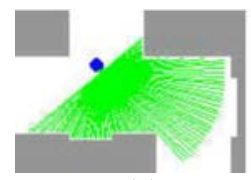

(a)

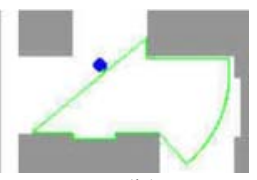

(b)
Fig.3. Representations of Laser Data

Figure 3-(a) represents laser range data along scan angle of a laser range finder and Figure 3-(b) shows the connection of detected vertex points following the detecting sequence. These two different representations use the same laser data. They have the same information about environments within detectable range but the numbers of data of the two are different. The number of data in Figure 3-(a) is same as the number of laser data, while the information in Figure 3-(b) can be described by some feature points and/or contour information whose number of data is much less than the number of raw laser range data. Therefore, if we are able to extract effective features to describe the raw laser data, the number of data can be decreased. For example, we can extract 7 lines from Figure 3-(b). It is, however, not easy and robust to extract line data since the number of lines can be varied according to the location of the laser range finder for measurement and sometimes we cannot detect short lines due to sensor noise. Furthermore, the process for line extraction from laser data includes much computational loads to use a robust algorithm such as Generalized Hough Transform. So, we adopt anglehistogram as an effective feature to describe the laser range data.

\subsection{Angle-Histogram}

In [8], angle-histogram is introduced. When we interpret each laser range as a vector, the difference between consecutive vectors can be calculated. The frequency of the occurrence of each vector difference with respect to each angle of vector difference is called angle-histogram. To find an effective anglehistogram for a set of range data, we do not use laser data whose range is maximum because these laser data are represented as an arc in $x-y$ plane and angle difference in the arc region has little influence on overall angle-histogram. Figure 4 shows an example of angle-histogram for laser data in Figure 3. If there is no error in laser data, we can obtain an ideal angle histogram shown in Figure 4-(a). However, there are sensor noises in real laser data and its anglehistogram from these inaccurate laser data does not have these simple patterns. To solve this problem, we filtered noisy laser data by low-pass-filtering method for obtaining similar pattern to ideal angle-histogram. A filtered angle-histogram obtained from noisy laser data is shown in Figure 4-(b).

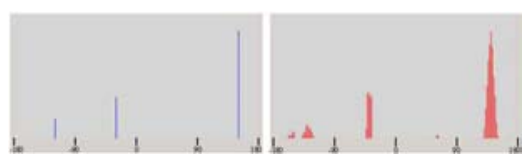

Fig.4. Angle-histogram for Laser Data in Fig. 3 (a) Ideal Angle Histogram, (b) Filtered Real Angle-Histogram

The main advantage of using angle-histogram is that we can reduce computational loads and processing time for localization, and we can find the heading angle of the mobile robot fast because the anglehistogram includes information on the slope and length of detected environments even though laser data has sensor noises as shown in Figure 4-(b). Of course, angle-histogram has disadvantages considering that there can be same angle-histograms even when obtained laser data are quite different because this method simplifies laser range data. However, we can overcome this problem by Suitable-for-ML(SFML) angle-histogram.

\section{Localization Using Angle-Histogram}

\subsection{Basic Concept of ML using Angle-Histogram}

The update part of ML is updating the probability of each state using sensor model by laser range data and desired range data of each state. On the other hand, we update the probability of each state using our 
sensor model by the length differences between reference and obtained histogram. Figure 5 shows the comparison of our method with ML.

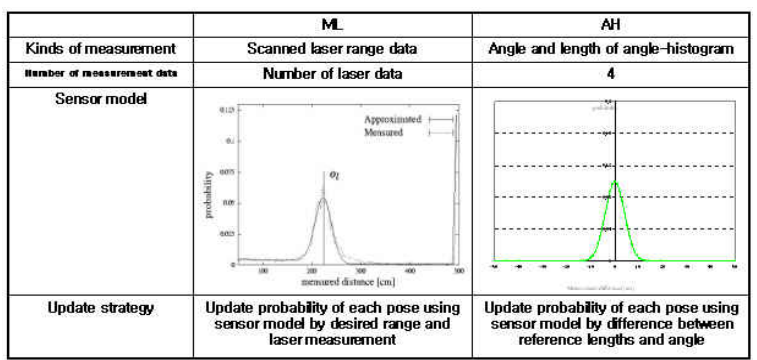

Fig.5. Comparison Our Method with Markov Localization

The update part follows the following steps. At each time step $T$, we obtain laser data and calculate modified angle-histogram. For each state $L_{T}$, we compare lengths of local maximum points in reference with lengths of corresponding angle in obtained histogram. Using this method, we can reduce the quality of calculation to $4 /$ (number of laser data). Of course, this method has disadvantages: inaccurate length of a obtained histogram, and many similar data in references. And, we cannot reduce comparative states. We only reduce comparative measurement data.

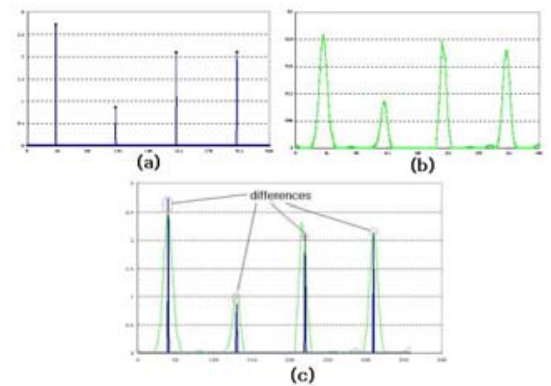

Fig.6. (a) Reference Angle-Histogram, (b) Obtained AngleHistogram, (c) Length Differences Used in the Update Part

\subsection{Probability update based on the SFM Angle- Histogram}

\section{1) Obtaining method of exact length of histogram}

If we look over a line made from laser data, we can see that it is composed of many lines because of sensor noises as shown in Fig.7. This phenomenon makes the length of angle-histogram inaccurate. In the basic angle-histogram, we obtain the length of histogram just by summing length of vectors that have same angle. However, this length can vary according to noises. Therefore we have to obtain the exact length of histogram. Let the line equation of Fig. 7 to be $y=a x+b$. Angle difference between line of $l_{i}$ and line $y=a x+b$ is $\theta_{i}-\tan ^{-1}(a)$. Therefore, we can obtain the exact length of histogram by

$$
\text { real length of histogram }=\sum_{i=1}^{n}\left[l_{i} \times \cos \left(\theta_{i}-\tan ^{-1}(a)\right)\right]
$$

where $n$ is the number of lines.

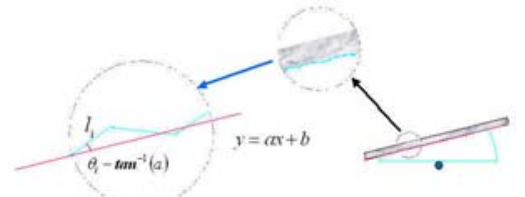

Fig.7. Obtaining Method of Exact Length in Histogram

\section{2) Discriminating method among similar patterns}

As we mentioned in section 3, there can be many similar patterns in reference because we simplify many laser data into angle-histogram. To distinguish correct state from a lot of similar patterns, we use an additional reference. We use one minimum range at each state regardless of detecting angle of this range.

\section{3) Reduction of comparative state}

We reduce comparative measurement data by anglehistogram. However, if we also can reduce comparative states, calculations are drastically reduced. We reduce comparative states (especially comparative angle) by the characteristics of anglehistogram under the assumption that large parts of the obstacles are located parallel to walls. In the corridor of most buildings, walls are placed in orthogonal. In such orthogonal environments, the angle-histogram becomes more powerful than in common environments due to the special characteristics of angle-histogram. Figure 8-(a) is an example of orthogonal environments, and Figure 8(b) is angle-histogram of them. In this anglehistogram, we can divide it into 4 parts according to the 0 -to-90 degree range area. Part (1) of Figure 8-(b) means the detected length and angle of environment (1) of Figure 8-(a). And environments of Figure 8-(a) are placed in orthogonal. Therefore, we can write each angle and length of Figure 8-(b) as

$$
\begin{aligned}
& <\theta_{1}, l_{1}> \\
& <\theta_{2}, l_{2}>=<\theta_{2}, l_{2}>=<\theta_{1}+1 \times 90, l_{2}> \\
& <\theta_{3}, l_{3}>=<\theta_{3}, l_{3}>=<\theta_{2}+1 \times 90, l_{3}>=<\theta_{1}+2 \times 90, l_{3}> \\
& <\theta_{4}, l_{4}>=<\theta_{4}, l_{4}>=<\theta_{3}+1 \times 90, l_{4}>=<\theta_{1}+3 \times 90, l_{4}>
\end{aligned}
$$

In those equations, we can regard each angle as

$$
\theta_{i}=[90 \times(i-1)]+\theta_{1}, \quad \text { where } i=2,3,4
$$

Then, if we sum each part of Figure 8-(b) regardless of added 90 degrees(shown in Figure 8-(c)), then we can obtain a pattern of Figure 8-(d). In Figure 8-(d), we can obtain maximum angle $\theta_{a}$ and error boundary $\varepsilon$. This means that the range of heading angle of the robot is

$$
\left(\theta_{a}-\varepsilon\right)+(90 \times i) \leq \text { heading } \leq\left(\theta_{a}+\varepsilon\right)+(90 \times i)
$$

where $i=0,1,2,3$. We can use this equation under our environmental assumption. Also, we obtained similar results when we use $1^{\circ}$ angle resolution or $4^{\circ}$ angle resolution. Therefore, we can also enlarge the angle resolution to $4^{\circ}$. 
When we use the length of four local maximum points in the SFML angle-histogram and one minimal range, $N_{\text {calculation }}$ becomes

$$
\begin{aligned}
N_{\text {calculation }} & =N_{x^{\prime}} \times N_{y^{\prime}} \times N_{\theta^{\prime}} \times N_{\text {data' }} \\
& =N_{x} \times N_{y} \times\left[4 \times\left(N_{\theta} \times \frac{2 \varepsilon}{360}\right)\right] \times\left(N_{h}+N_{m}\right) \\
& =N_{x} \times N_{y} \times 10 \times \varepsilon
\end{aligned}
$$

where $\left\{\begin{array}{l}\varepsilon: \text { range of possible heading area } \\ N_{h}: \text { number of peaks in histogram }(=4) \\ N_{m}: \text { number of minimum range }(=1) \\ N_{\theta}: \text { number of comparative angles }\left(=\frac{360}{\text { angle resolution }\left(-4^{\circ}\right)}=90\right)\end{array}\right.$

If we use $300 \times 400$ grid-map with $2^{\circ}$ angle resolution and the number of laser data is 181 , then we have to perform 3,909,600,000 calculations when we use ML. If error boundary is $\varepsilon= \pm 10$, our algorithm has to perform $12,000,000$ calculations. That is, we can reduce calculation as $1 / 325$ in theoretical results.

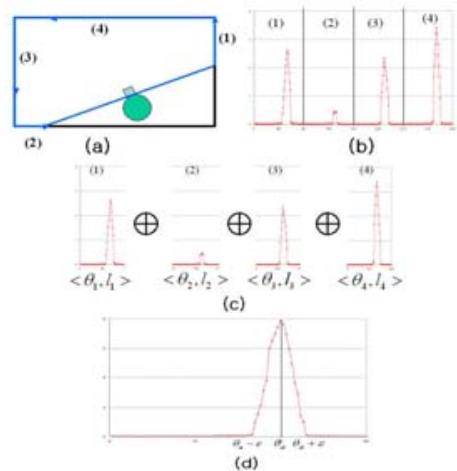

Fig.8. Reducing Method of Comparative States

\section{Experimental Results}

\subsection{Simulation Results}

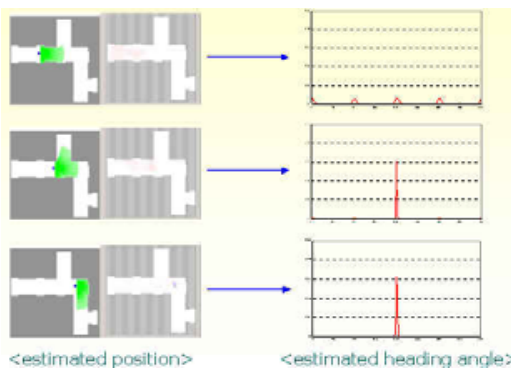

Fig. 9. Simulation Result of Pose Estimation of Basic Angle-Histogram while Robot Moving

We used a personal computer equipped with Pentium-III 700Mhz MPU with 128M RAM. The test-bed for simulation is a mobile robot named KARA developed in our laboratory for experiments. The mobile robot has two differential wheels, and is equipped with a SICK laser range finder that covers frontal half of the robot. Total number of data for each laser scan is 361 when its scan resolution is 0.5 degree and its maximum measurable distance of the laser range finder is set to 4 meters. For simulation, we added white Gaussian noises to laser range data.
Environments are set as the corridor including our laboratory.

Figure 9 shows simulation results of basic form of angle-histogram. In this simulation, the physical size of environments is $15 \times 15(\mathrm{~m})$ and we make $75 \times 75$ (each grid size is $20 \times 20(\mathrm{~cm})$ ) grid-map with $1^{\circ}$ angle resolution. The mobile robot moves while obtaining the laser range data. We set the initial probabilities of all state to be uniform distribution before starting these localization steps. Left side of Figure 9 is the probability of estimated position and right side is the probability of estimated heading angle. The probabilities of many states seem to have similar values because there are many similar patterns. Even though the position probabilities are not focused on the position where the mobile robot really exists, we can obtain the heading angle of 0 degree in that step. It is noted that the position error is maintained within an error bound when a mobile robot knows the exact heading angle while $x-y$ position includes some errors. And, if a mobile robot knows the exact $\mathrm{x}-\mathrm{y}$ position and wrong heading angle, the position error will be increased during the mobile robot is moving. Therefore, our proposed approach will reduce the location error resulted from the error in heading angle of a mobile robot.

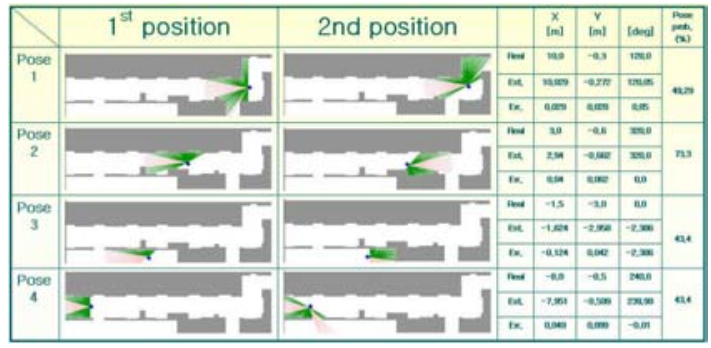

Fig. 10. Simulation Result of Pose Estimation of SFM Angle-Histogram while Robot Rotating

Figure 10 shows simulation results of SFML angle-histogram while robot rotating. In this simulation, the physical size of environments is $25 \times 7(\mathrm{~m})$ and we make $250 \times 7075 \times 75$ (each grid size is $10 \times 10(\mathrm{~cm}))$ grid-map with $4^{\circ}$ angle resolution. The average $\mathrm{x}$-error is $6.05 \mathrm{~cm}$, y-error is $5.77 \mathrm{~cm}$, and heading-error is 0.6 degree. We obtain exact pose when robot rotates. Note that we can obtain less than $1^{\circ}$ heading-error even though angle resolution is $4^{\circ}$.

\subsection{Experimental Results}

Figure 12 shows an experimental result of basic angle-histogram. The robot is moving from the point ' $S$ ' to the point ' $E$ ' and the localization is executed at circular points in the figure. A dotted circle at each localized point denotes the probable position area. We can see that the area of dotted circle is decreased as performing localization repeatedly. Even though the localization accuracy is decreased as heading angle of robot changes in many localization 
algorithms, it is possible to obtain more accurate position when heading angle is changed using our algorithm. Four local maximum points in anglehistogram are used to make a model for environments and to update the probability of each cell. So, the number of iterations for ML in each step is reduced from $125 \times 85 \times 180 \times 181$ to $125 \times 85 \times 180 \times 4$. The position accuracy is less than one grid cell while the rotational error is less than 2 degrees when we use basic angle-histogram.

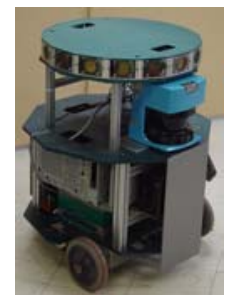

Fig. 11. Mobile Robot KARA for Experiments

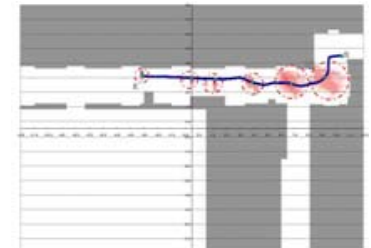

Fig. 12. An Experimental Result of Basic Angle-Histogram

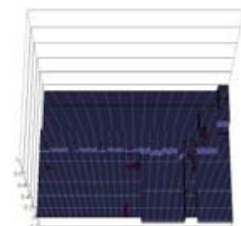

(a)

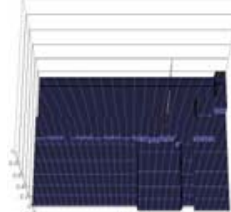

(d)

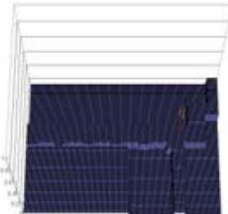

(b)

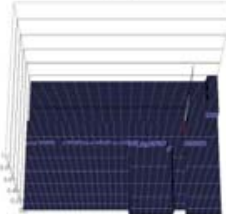

(e)

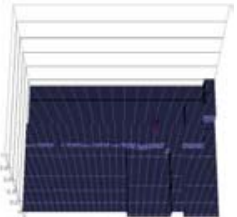

(c)

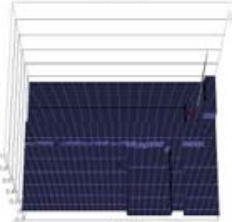

(f)
Fig.13. Experimental Results of SFM Angle-Histogram

Figure 13 is an experimental result of SFML anglehistogram. In this experiment, we swept off other obstacles like cabinets and bookshelves to make environments simpler than those of basic anglehistogram. Robot moves from left to right, and turns to return the starting point. We can see that state probabilities of Figure 12 are converged to real position faster than Figure 12 nevertheless of the simplicity of environments. We also can see that the converged state probability becomes nearly $90 \%$ after turning. This means that SFML angle-histogram makes better result when robot changes its heading.

\section{Conclusions}

We have proposed a fast global localization algorithm via sensor data remodeling using angle histogram. Remodeled comparative measurement data using angle histogram for a set of range data is used instead of real range data. By applying the angle histogram for the measurement modeling in estimation process, we can reduce the number of comparative measurement data and computational loads dramatically. Also, we can obtain the heading angle of the mobile robot exactly in a very fast manner. So, the proposed approach will help to reduce navigation errors resulted from the heading errors of a mobile robot.

Future work is concerned on the complex environments that have many patterns. And, sensor data fusion will be investigated to reduce the iteration for position convergence.

\section{REFERENCES}

[1] Patric Jensfelt and Henrik I. Christensen, "Laser Based Pos Tracking", Proceedings of IEEE International Conference on Robotics and Automation, pp. 2994-3000, 1999.

[2] Sooyong Lee, Nancy M. Amoto and James Fellers, "Localization based on visibility sectors using range sensors", Proceedings of IEEE International Conference on Robotics and Automation, pp. 3505-3511, 2000.

[3] Joachim Weiber, Klaus-Werner Jorg and Ewald von Puttkamer, "APR-Global Scan Matching Using Anchor Point Relationships", Proceedings of International Conference on Intelligent Autonomous Systems, Venice, Italy, pp.25-27 2000.

[4] Dieter Fox, "Markov Localization: A Probabilistic Framework for Mobile Robot Localization and Navigation", Ph.D. Thesis, University of Bonn, Germany, 1998.

[5] Frank Dellaert, Dieter Fox, Wolfram Burgard and Sebastian Thrun : "Monte Carlo Localization for Mobile Robots", Proceedings of IEEE International Conference on Robotics and Automation, Vol. 2, pp. $1322-1328,1999$.

[6] Leopoldo Jetto, Sauro Longhi and Giuseppe Venturini, "Development and experimental validation of an adaptive extended Kalman filter for the localization of mobile robots", IEEE Tansactions on Robotics and Automation, Vol. 15, No. 2, pp.219229, April, 1999.

[7] Jung-Hyun Moon, Bum-Jae You, Hagbae Kim and Sang-Rok Oh, "Fast Markov Localization using Angle-Histogram", Proceedings of IEEE International Conference on Advanced Robotics(ICAR), vol.1, pp.411-416, June, 2003.

[8] Gerhard WeiB, Christopher Wetzler, Ewald von Puttkamer, "Keeping Track of Position and Orientation of Moving Indoor Systems by Correlation of Range-Finder Scans", Proceedings of IEEE International Conference on Intelligent Robots and Systems, pp. 595-601, Sep. 1994. 